\title{
INTRODUCIENDO LOS TRABAJOS ARTESANALES EN LA EDUCACIÓN INFANTIL: LA TARACEA GRANADINA COMO RECURSO ETNOMATEMÁTICO
}

Boada Rafecas, Nuria; Fernández-Oliveras, Alicia; Oliveras Contreras, María Luisa. Universidad de Granada.

Fecha de recepción: 30 de junio de 2014.

Fecha de revisión: 5 de julio de 2014.

Fecha de aceptación: 15 de julio de 2014.

\section{Resumen}

Este trabajo supone un acercamiento a las matemáticas desde el enfoque de las etnomatemáticas, la cultura y el método didáctico por proyectos, con el que planteamos un caso práctico para abordar la didáctica de las matemáticas en el segundo ciclo de educación infantil. En primer lugar se trata la fundamentación teórica, siguiendo la línea de investigación educativa. En ella se abordan los conceptos de matemáticas (como ciencia, manifestación cultural y forma de pensamiento), etnomatemáticas (las matemáticas desde las distintas perspectivas culturales), cultura (como condicionamiento contextual en la interpretación de las matemáticas), y método por proyectos (como metodología didáctica capaz de generar aprendizaje significativo desde contextos particulares). Seguidamente se plantea un caso práctico que se apoya en el método de aprendizaje basado en proyectos. La propuesta se describe en tres apartados, -marco conceptual, fundamentación y programación-, y responde a un tipo de diseño curricular denominado microproyecto. El objeto de estudio elegido para el microproyecto es la taracea, una técnica artesanal típica de Granada, basada en la combinación matemática de formas geométricas. El desarrollo de este microproyecto persigue trabajar las matemáticas desde una perspectiva más cercana a la infancia y facilitar un aprendizaje basado en el constructivismo.

Palabras clave: Constructivismo social, matemáticas, educación intercultural, aprendizaje basado en proyectos, estrategia globalizadora, formación de profesores.

El presente trabajo versa entorno a las matemáticas y qué significado tienen dentro de la Cultura de nuestro entorno. Concretamente se presenta la Taracea como objeto de estudio elegido; una artesanía con gran arte popular dentro de la cultura andaluza.

Sin pretensión de enfocar el trabajo de una manera generalizada sino sintetizando al máximo, debido a la amplitud y cantidad de posibilidades que existen para trabajar las matemáticas, nos centramos en aspectos concretos e interesantes de esta nueva corriente filosófica-epistemológica, como es la Etnomatemática y la influencia de la cultura en su diversidad de representaciones.

El concepto matemáticas despierta muchas sensaciones, para muchos de nosotros nos recuerda a algo abstracto, aburrido y difícil, algo a lo que se tiene aversión y que solo utilizamos en la escuela para tener un aprobado. Esto también está relacionado con una manifestación únicamente formal, con un enfoque exclusivo de la matemáticas que impide que los/as alumnos/as aprendan a partir de sus experiencias; 
influenciados por un sistema educativo que todo lo mide, pensamientos negativos, malas experiencias y el preguntarse cuestiones como: ¿qué hago cuando ya lo he aprendido?, ¿para qué me sirve y como lo aplico en mi vida diaria?

Un currículo como el nuestro, donde el aprendizaje se convierte en aprender métodos correctos de resolución, y que está orientado hacia una técnica que promueve el hacer instructivo más que un hacer interpretativo, son ejemplos que han de inquietar los bastante como para plantear cambios.

Las matemáticas no significan solo la realización de operaciones aritméticas, es mucho más que todo esto. Se trata de entenderlas como un producto cultural, una forma de pensamiento y de lenguaje que nos permite comprender el entorno donde vivimos. Por eso las matemáticas son propias de cada cultura.

Pero entonces, ¿que son las matemáticas? En el siguiente apartado tratamos de dar una explicación en torno a este concepto y que metodología puede resultar más eficaz para su aprendizaje.

\section{FUNDAMENTACIÓN}

Las matemáticas son una ciencia, un lenguaje, una herramienta de cálculo... Definir el concepto "matemáticas" de forma precisa, es difícil debido a su carácter tan amplio. Para ello nos centramos en una definición que clarifica a modo de metáfora los tres puntos de vista desde los que se puede considerar las matemáticas: Las matemáticas son como un trébol, con una hoja que las caracteriza como ciencia, otra hoja indica que son una manifestación cultural inseparable del resto de los aspectos de cada cultura y la tercera hoja simboliza que son parte de nuestra forma de pensar y de concebir el mundo (Oliveras 2000, p.46).

Por tanto, las Matemáticas se caracterizan por varios aspectos relacionados entre sí, siendo al mismo tiempo una ciencia, una manifestación cultural y una forma de pensamiento, ya que todos pensamos matemáticamente en nuestra vida diaria, son una parte de nuestro pensamiento.

\section{¿Qué son las Etnomatemáticas?}

El tema que tratamos de clarificar a continuación es el significado de Etnomatemáticas. Para realizar un análisis sobre Etnomatemáticas y entender su significado, primero se hace necesario cuestionarse que significa dicho término. Seguramente a la hora de pensar su significado cada persona tendrá una forma distinta de interpretar y explicar la palabra "Etnomatemáticas", que dependerá de sus conocimientos y experiencias previas.

Para hacer una primera aproximación, y tratando de definir este concepto de una forma simple, se puede decir que las Etnomatematicas son las distintas formas de matemáticas propias de los grupos culturales. Sin olvidar que a la vez representan al conjunto de todas las matemáticas existentes.

En particular, cuando hablamos de matemáticas podemos decir que éstas tienen raíces culturales y de hecho son un sistema cultural. Por eso, debemos recordar que los niños y niñas llegan a la escuela con sus propias lógicas matemáticas. Cada uno/a tiene sus propios patrones de comportamiento, códigos, símbolos, modos de razonar, maneras de medir, clasificar y matematizar. Estas distintas lógicas matemáticas, propias de los diferentes grupos culturales, son las que llamamos Etnomatemáticas. 
El prefijo "etno" se refiere al entrono natural y cultural, a cualquier grupo de sociedades con características propias, tradiciones religiosas, clases profesionales...También incluye sus jergas, códigos, símbolos, mitos, y modos específicos de razonamiento e inferencia (D'Ambrosio, 1987, citado por Oliveras, 1996).

El término Etnomatemática fue acuñado por d'Ambrosio para describir las prácticas matemáticas de diferentes grupos culturales. Sus ideas y pensamientos propiciaron la aparición de una nueva corriente de pensamiento en la educación matemática, la que hoy entendemos como Etnomatemática. En su definición hace referencia a elementos vinculantes a la cultura: Nosotros llamaremos Etnomatemátícas, a las matemáticas que se practican entre grupos culturalmente diferenciables, como sociedades nacionales o tribales, grupos de trabajo, niños de un cierto tramo de edad, clases profesionales, incluyendo sus jergas y modos de expresión (D'Ambrosio, 1985, p. 45).

Desde un punto de vista estrictamente matemático, debemos ser conscientes de las consecuencias de esta nueva concepción:

1. La existencia de elementos comunes que caracterizan y configuran todas las matemáticas (como los procesos de clasificar, ordenar, contar y medir).

2. La existencia de desarrollos matemáticos, independientes de cada cultura, igualmente válidos.

3. La concepción de las matemáticas formales o escolares como uno de los muchos tipos de Etnomatemáticas, en absoluto el único o el mejor.

A lo largo de la historia tenemos constancia de que existen una gran cantidad de culturas, todas nos dan la posibilidad de realizar los distintos procesos matemáticos, contar, razonar, medir, representar, establecer relaciones y explicaciones simbólicas, pero todas ellas tienen un conocimiento común, que es el matemático. Entonces, ¿qué influencia tiene el lenguaje en las Matemáticas?

Un ejemplo relacionado con la geometría, que trata de ejemplificar que las matemáticas no son universales, es el de la definición de cuadrado. Muchas de las casas que se construyen en los poblados de Angola son de planta cuadrada, aunque para su construcción no necesitan contar cuatro lados ni comprobar su condición de lados paralelos. Solo les hace falta tener dos palos iguales y situarlos en forma de cruz en su punto medio. Así, los extremos de los palos serán las esquinas de la habitación. Con esto podemos observar como cada ser humano ha ido construyendo sus propias ideas y representaciones acerca de las matemáticas desde el contexto de sus grupos de pertenencia. Todo esto nos sirve para pensar y sentir más allá de nuestra visión euro-céntrica de las matemáticas. Aunque obtenemos lo mismo, el modo de pensarlo y construirlo es distinto.

Estos ejemplos nos muestran hasta qué punto la cultura y los distintos entornos culturales influyen sobre las prácticas matemáticas escolares. Esto es lo que nos conduce a hablar de cultura.

\section{¿Qué entendemos por cultura?}

La antropología como ciencia ha contribuido enormemente a la comprensión y estudio de las diferentes culturas. Existen muchas definiciones del concepto de cultura que han posibilitado, no solo el enriquecimiento de la teoría antropológica sino, el diálogo con otras ramas de conocimiento.

Clifford Geertz (1988) define la cultura como "ideas basadas en el aprendizaje cultural 
de símbolos y concibe las culturas como mecanismos de control: planos, recetas, reglas, construcciones-, lo que los técnicos en ordenador llaman programas para regir el comportamiento" (p.29).

Este autor es considerado el creador de la "antropología simbólica" que en contraste con la cultural se centra en la observación de las distintas realidades, es decir, en las diferentes maneras que la gente entiende su entorno. Todas estas interpretaciones se establecen por medio de símbolos y procesos, un claro ejemplo son los rituales; y es a través de ellos como los seres humanos establecen significados a sus acciones. Cada interpretación debe permitir analizar la cultura en busca de significados. Esto nos conduce a la existencia de una multitud de realidades en la que cada persona las interpreta de una forma distinta.

Si observamos nuestra sociedad nos daremos cuenta de que está repleta de distintas formas de entender las matemáticas, cada cultura desde su propia forma de pensamiento. En definitiva, se trata de una forma de lenguaje.

\section{Método didáctico por Proyectos}

¿Cómo podemos trabajar las matemáticas en el aula de Infantil? Las Etnomatemáticas responden a una metodología, a una manera de actuar y dinamizar el aula, a distintas formas de pensar y vivir la cultura propia de cada sociedad. Por lo que este concepto nos aboca a la idea de proyecto, a una metodología que no separe las distintas áreas de conocimiento, que nos permita organizar los contenidos curriculares bajo un enfoque globalizador y en la que el niño, sienta que las matemáticas tienen una conexión con su entorno más cercano.

Por eso, la mejor forma de transmitir matemáticas es a través del Método por Proyectos. Esto nos remite a que el enfoque educativo tradicional se presenta en numerosos artículos e investigaciones como un método erróneo para lograr un correcto proceso de enseñanza-aprendizaje, pues, como mencionan Requesens y Díaz (2009), desde el punto de vista metodológico, la suficiencia en el dominio y la exposición por parte del profesor de los conocimientos disciplinares, no resuelve el déficit generado por la cultura contemporánea en cuanto a la capacidad de pensar, de organizar racionalmente la información, de buscar su sentido y, en consecuencia, de analizar críticamente la realidad.

Se necesita, por tanto, un nuevo enfoque didáctico, desde la manera en que el profesor imparte su clase hasta la forma en que el alumno adquiere el conocimiento. Según Aebli (1979), una teoría que logra adaptarse fácilmente a este nuevo contexto es el constructivismo. Según Carretero (1997): El constructivismo es la idea que mantiene que el individuo, -tanto en los aspectos cognitivos y sociales del comportamiento como en los afectivos- no es un mero producto del ambiente ni un simple resultado de sus disposiciones internas, sino una construcción propia que se va produciendo día a día como resultado de la interacción entre esos dos factores (p. 50).

En esta teoría cognitiva constructivista se fundamenta el Método por Proyectos, cuyos precursores son William Heard Kilpatrick y John Dewey, partidarios de una enseñanza centrada en el niño. Este planteamiento didáctico tiene como base el desarrollo de un conocimiento globalizado y relacional.

Hernández (1988) ha definido esta estrategia organizativa como una forma de organizar la actividad de enseñanza/aprendizaje en la clase, que implica asumir que los conocimientos escolares no se articulan para su comprensión de una forma rígida, 
en función de unas referencias disciplinares preestablecidas, y de una homogeneización de los individuos y de la didáctica de las disciplinas. Por ello, la función del proyecto de trabajo es la de crear estrategias de organización de los conocimientos basándose en el tratamiento de la información y el establecimiento de relaciones entre los hechos, conceptos y procedimientos, que facilitan la adquisición de conocimientos (p. 55).

Por tanto, el aprendizaje basado en proyectos es una estrategia de enseñanzaaprendizaje en la que, tanto la adquisición del conocimiento, como el desarrollo de las habilidades y actitudes resulta importante.

Para que pueda ser llevada en el aula de Educación Infantil, esta metodología ha sido adaptada por Oliveras (2006) con enfoque etnomatemático y dándole el nombre de "microproyecto" definiéndola como: Un diseño curricular de núcleos de conocimiento interdisciplinar que surge de la Etnomatemática. Partiendo de un "signo cultural" con potencial matemático, el equipo docente reflexiona, activando el descubrimiento de "Matemáticas Situadas" en contextos culturales, e implica el rol investigador en los profesores (p. 125).

En síntesis, podemos decir que Etnomatemáticas es un concepto que va mucho más allá de las matemáticas ya que engloba otras formas de conocimiento como el arte, la religión, la música, las ciencias... Su repercusión en el aula de Educación Infantil es importante y presenta una forma operativa de llevar al aula el constructivismo mediante el Método por Proyectos y el enfoque micro-referido, es decir, a través de un microproyecto. $\mathrm{Y}$ es esto es lo que en esencia se plantea en el presente trabajo: la elaboración teórica de un microproyecto.

\section{DISEÑANDO UN MICROPROYECTO}

El diseño de un microproyecto para el segundo ciclo de educación infantil requiere un proceso que consta de varias fases. La primera es seleccionar un objeto de estudio interesante y con potencial de conocimientos variados. El objeto de estudio elegido se encuentra dentro de un campo artesanal y es la Taracea. En este sentido, se presenta el estudio de la taracea como un "arte popular" de la Cultura Andaluza, como una técnica específica con un gran significado cultural.

Para la recogida de información y datos entorno a esta artesanía, las técnicas utilizadas han sido la observación directa y una entrevista no estructurada a un artesano de la taracea, en la que se ha recopilado información acerca del proceso de producción del objeto, su historia y la evolución de la técnica utilizada. Posteriormente, se ha realizado un análisis documental teórico para descubrir el mundo de la taracea, la relación con aquellos aspectos matemáticos, según el amplio concepto de "Etnomatemáticas", por el que se desprenden los conceptos que se expondrán a continuación.

\section{Concepto de Taracea}

La taracea constituye una parte fundamental y determinante del acervo patrimonial y cultural de esta región. Esta técnica empezó a realizarse en los pueblos árabes, pero con la llegada del Islam a Oriente la Taracea se llenó de simbolismo y belleza. Un arte introducido por los árabes en España que enseguida se asocia al monumento más emblemático de esta región: La Alhambra.

En la mayoría de los casos se realiza de forma manual e individual. La combinación de 
distintos tipos y tonos de madera y el conjunto de elementos combinados reflejan el estilo y el respeto por la autenticidad de la obra. Debe respetarse siempre el diseño geométrico, pero la forma de colocar las piezas es particular del artesano, por lo que su originalidad siempre estará en el diseño. Aunque la creatividad y originalidad del artesano existe, está limitada; creemos que se ha convertido en hábito, al haberse estandarizado, ya que los aspectos matemáticos que se utilizan son básicamente los giros, la simetría y las traslaciones, para la construcción de estrellas y grecas.

Su técnica consiste en la incrustación. La variedad de colores y el tipo de material utilizado provoca un efecto de contraste entre unas piezas y otras que dan como resultado la Taracea. Para su elaboración el material empleado son distintos tipos de madera como es el olivo, ébano, el limoncillo o la madera de coral...y en algunas piezas se incrusta algo de metal como bronce o plata. La parte de formas geométricas tan idénticas como son las estrellas, se hace encolando de dentro a fuera unas varillas de diversos materiales como el hueso, la madera y el metal; estas varillas tienen forma de rombos, cuadrados o rectángulos, en su sección transversal; una vez terminado el bloque unido por varias varillas y pegado con cola se corta en rodajas a como si fuera un "salchichón", cada "rodaja" es denominada estrella.

Este arte se mantiene fiel a sus orígenes, los materiales y sus diseños son los mismos que se empleaban en los s. XIV y XV, solo su acabado admite innovaciones. Antiguamente se terminaba todo a mano con laca, pero actualmente las piezas de uso como mesas, bandejas... se terminan con poliuretano.

Según el Diccionario de la Real Academia Española, Taracea es un vocablo de origen árabe que significa "embutido hecho con pedazos de madera en sus colores naturales, o de madera teñida, concha nácar y otras materias". Sin embargo, la palabra más conocida para esta técnica es una de origen francés, "marquetería".

\section{Concepto de artesanía}

Para abordar el concepto de artesanía, nos centraremos en una definición de Fernández (2003) que engloba a otros tipos de artesanía (como las folklóricas, tradicionales, etnográficas, etc.) y que tiene en cuenta la realidad de la misma en la actualidad:

Actividad productiva y creativa de carácter plástico manual e inspiración tradicional; de confección y planificación seriada; que se materializa en objetos...o piezas que responden a una necesidad funcional o de uso cotidiano, decorativo, mágico...; facturada en un taller doméstico o profesional reducido, sin procesos tercerizados; mediante técnicas manuales y no manuales; cuya producción es restringida y destinada a un mercado reducido, de ferias o comercial pero conocido por el artesano.

El concepto de artesanía lleva intrínseco un valor económico, social y cultural debido a la importancia de dónde está hecho, quién lo ha hecho y como lo ha hecho ( $p .25)$.

\begin{tabular}{|l|l|}
\hline Económico & $\begin{array}{l}\text { Actividad económica generadora de } \\
\text { riqueza } \\
\text { Desarrollo de la economía local }\end{array}$ \\
\hline Social & Tradición productiva y artística \\
\hline Cultural & \\
\hline
\end{tabular}

Tabla 1. Valores intrínsecos de la artesanía. 
A lo largo del tiempo este concepto ha adoptado diferentes definiciones. Su definición actual es bastante heterogénea y polémica en función de su perspectiva:

- Tecnológica.- Su definición es de carácter fundamentalmente manual y con cierto sentido artístico.

- Antropológica.- La tradición es la que asigna a estos productos una función dentro de la comunidad. Desde esta perspectiva, cabe entender por artesanía toda actividad, retribuida o no, que no haya sido afectada por los principios de especialización, división y mecanización del trabajo.

- Cultural.- El concepto artesanía se funde con el concepto de "arte popular" entendido como aquel conjunto de actividades productoras, de carácter esencialmente manual, realizado por un solo individuo o una unidad familiar, trasmitidas de padres a hijos y cuyos productos, generalmente de carácter anónimo, están destinados a la cobertura de necesidades concretas.

\section{Justificación}

La recopilación de información entorno a la Taracea y su posterior análisis, ha permitido tener un conocimiento más cercano del perfil matemático que lleva intrínseco esta artesanía y de cómo el proceso de producir un objeto mediante la taracea puede ser efectivo en un aula de Educación Infantil mediante una adaptación escolar. Así la decisión de trabajar la Taracea en un aula de 5 años, se ha realizado teniendo en cuenta las características cognitivas de los niños.

La introducción de actividades basadas en esta artesanía hace posible plantear cuestiones matemáticas de aquellas cosas que nos rodean en nuestro entorno físico más cercano; permite crear una relación entre la experiencia de los alumnos y el conocimiento matemático. No obstante, reúne una variedad de contenidos y posibilidades didácticas que se pueden relacionar entre sí. El aprendizaje que supone la simulación de ser un artesano, va desde la adquisición de conceptos geométricos, topológicos (plano-espacio), coordinación óculo-manual y psicomotriz, hasta el desarrollo de la creatividad; por lo que no se aparta de los contenidos curriculares. Asimismo, existe una analogía entre los artesanos y los niños, ya que el pensamiento del artesano tiene cierta semejanza con el de los niños. La Taracea está muy cercana al mundo del niño, atañe al arte y a la belleza, a la parte estética de la cultura.

Existen otros aspectos que demuestran la validez de trabajar esta artesanía en el segundo Ciclo de Educación Infantil, más concretamente en 5 años. El pensamiento y la práctica de las matemáticas están en el uso cotidiano de todos, pero no siempre somos capaces de reconocerlas fuera del contexto escolar, de ahí la importancia de trabajarlas en contextos de la vida cotidiana, para acercarse de una forma más amena y creativa a la pluralidad de las matemáticas.

Como refleja Alsina (2011): Un enfoque globalizado de la Educación Matemática en las primeras edades y el uso de contextos de la vida cotidiana para aprender matemáticas -o cualquier otro tipo de contexto de aprendizaje- no contribuyen por ellos mismos al desarrollo de la competencia matemática, sino que ello depende de cómo los profesionales planteamos y gestionamos las actividades (p.218).

Por otro lado, otro aspecto a considerar es que la Taracea cumple una secuencia didáctica constructivista, de acción-reflexión y representación. El niño/a desarrolla la acción mental a través de la observación, manipulación y experimentación del objeto, este conocimiento físico se conectará posteriormente con la reflexión, donde el niño toma las decisiones, pensando lo que quiere hacer. $Y$ finalmente representa el objeto, 
para explicar y simbolizar su pensamiento. A modo de síntesis, se trata de una secuencia donde el niño observa y hace, piensa y comunica.

De esta forma se pretende crear un entrono de aprendizaje basado en una artesanía típica de nuestra región como es la taracea.

\section{PROGRAMACIÓN}

Este microproyecto está diseñado para niños del segundo ciclo de Educación Infantil, concretamente para la edad de 5-6 años. En este apartado se analiza el proceso de producción del objeto de estudio y se delimitan los pasos a seguir. Para ello, se hace necesario realizar de un modo más exhaustivo la secuencia de las tareas de esta actividad artesanal. Es decir, un análisis de todo lo que conlleva el proceso físico de realizar un lapicero de Taracea, con la idea de determinar las habilidades matemáticas aplicadas, sensoriales y motoras necesarias para simular la actividad del artesano.

\section{Objetivos}

Objetivos generales:

- Conocer, a través de las matemáticas, una tradición del arte popular de la Cultura Andaluza como es la Taracea.

- Comprender el proceso de elaboración de un producto artesanal.

Objetivos específicos:

- Reconocer las distintas formas geométricas.

- Realizar seriaciones.

- Desarrollar la psicomotricidad fina.

- Reconocer los movimientos en el plano: simetría y giros (con las estrellas) y traslaciones (con las grecas), así como las formas planas y tridimensionales.

- Desarrollar la creatividad.

- Reflexionar y argumentar el proceso que se está realizando.

- Ampliar el vocabulario en torno a la artesanía de la Taracea.

- Experimentar la representación y expresión artística mediante el empleo de diversas técnicas.

- Conocer las propiedades topológicas.

\section{Contenidos}

- Reconocimiento de las formas geométricas: cuadrado, triángulo, rombo y rectángulo.

- Realización de seriaciones con los rotuladores de colores.

- Utilización de la lengua oral para argumentar el proceso que se está llevando a cabo en la elaboración del objeto.

- Ampliación del vocabulario en torno a la Taracea. Glosario de términos que aparecen: grecas, estrellas, taracea, tipos de madera como el olivo, ébano, el limoncillo o la madera de coral.

- Identificación de las formas planas y tridimensionales en los elementos del entorno.

- Expresión plástica a través de la armonización de colores. Colores básicos: rojo, verde, blanco, junto con colores oscuros como el marrón o el negro.

- Reconocer las propiedades topológicas: dentro, fuera. 


\section{Actividades}

Trataremos de jugar a ser artesanos, es decir, que los niños/as se conviertan en artesanos simulando su labor, aunque la secuencia de tareas no se tome igual que la que realiza el artesano debido a la complejidad manipulativa que requiere. Para ello, se propone una serie de actividades adaptadas al nivel de edad del alumnado. La secuencia de dichas actividades se esquematiza en la Figura 1.

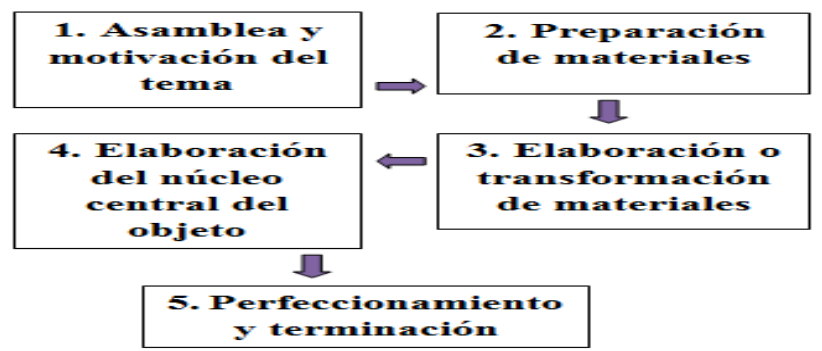

\section{- Asamblea y motivación}

En primer lugar, al ser una propuesta de tipo globalizado, es imprescindible que el niño se familiarice con el entorno de la artesanía, no solo como un medio para aprender matemáticas, sino como un instrumento para acercarse a la labor del artesano y poner en marcha todas sus capacidades. Por tanto, es necesario tener en cuenta que cualquier acción que realizan los niños (mirar un cuento, construir una torre, comer, dibujar, charlar con los amigos) pone en juego distintos ámbitos de su persona, el aprendizaje en estas edades no responde a "compartimentos estancos". Por lo que no es adecuado la presentación de esta artesanía en parcelas epistemológicas (matemáticas, lengua, ciencias...).

El primer contacto entorno a la taracea, es una tarea que depende en gran parte del profesor; la forma de presentar la propuesta y como esta les llegue a los niños/as será esencial para su motivación e interés. Por tanto, para que el niño se familiarice con este ámbito es necesario también identificar los conocimientos previos de los alumnos. Esto nos permitirá tener un punto de partida para organizar las actividades y detectar el nivel de dificultad que puedan tener. De igual modo, se podrá conocer el lenguaje de los alumnos y el contexto en el que se desenvuelven. Hay que tener presente que puede surgir la posibilidad de que los conocimientos previos que tengan los niños/as entorno a la Taracea sean muy escasos, en cualquier caso se introducirán nuevos elementos conceptuales para dotar de significado a los conceptos propuestos.

Una vez emprendido el microproyecto, donde el maestro/a presenta el tema, mediante un cuento, con imágenes audiovisuales o con algún objeto de taracea, se debate y se pide a los alumnos que traigan información. Para ello será necesario realizar una búsqueda en la biblioteca de la clase y en sus casas. Una vez se ha puesto en común en el aula se realizará una visita a un taller de Taracea, para que puedan ver de primera mano todo el proceso de fabricación de un producto y preguntar las cuestiones planteadas previamente en el aula, así como las posibles dudas que vayan surgiendo en el momento.

\section{- Actividades previas a la elaboración del objeto}

Para trabajar la simetría colocamos un espejo en dibujos que tengan simetría (una casita, un pino o una mariposa) y en otros que no la tengan (un coche, un gato o un 
perro):

- Observa que algunos no cambian.

- Encuentra otros dibujos que no cambien.

- Encuentra otros dibujos que cambien.

Pintamos con pintura la parte de la "estrella" de la Taracea. Después doblamos el papel para que se calque en el otro lado del papel y ver así su efecto simétrico.

\section{- Actividades para elaborar el objeto: Somos artesanos}

- Compra y preparación del material: ¿Qué necesitamos para hacer un lapicero de taracea (objeto)? cartulina, tijeras, pegamento, papel para plastificar, rotuladores y una tapa de plástico grande (por ejemplo, de un tarro de crema de cacao), que nos servirá de base. El alumnado elegirá el color de la cartulina que quieren para el lapicero.

- Elaboración o transformación de materiales: Para elaborar el material empezaremos la tarea trabajando en el plano para posteriormente pasarlo al espacio. De esta forma nos facilitará su ejecución, ya que trabajar un objeto relativamente pequeño desde una perspectiva tridimensional puede resultar más complejo en estas edades.

Se empezará repartiendo una cartulina con forma de rectángulo. Para el diseño y decoración del lapicero se les darán distintos modelos de grecas y estrellas (Figura 2) con dos tamaños, pequeño y grande. La tarea aquí consiste en pensar el diseño, la organización de los elementos en el plano, darle color a las grecas y estrellas mediante seriaciones, delimitar el tamaño de estas y recortarlas para adecuarlas al plano.
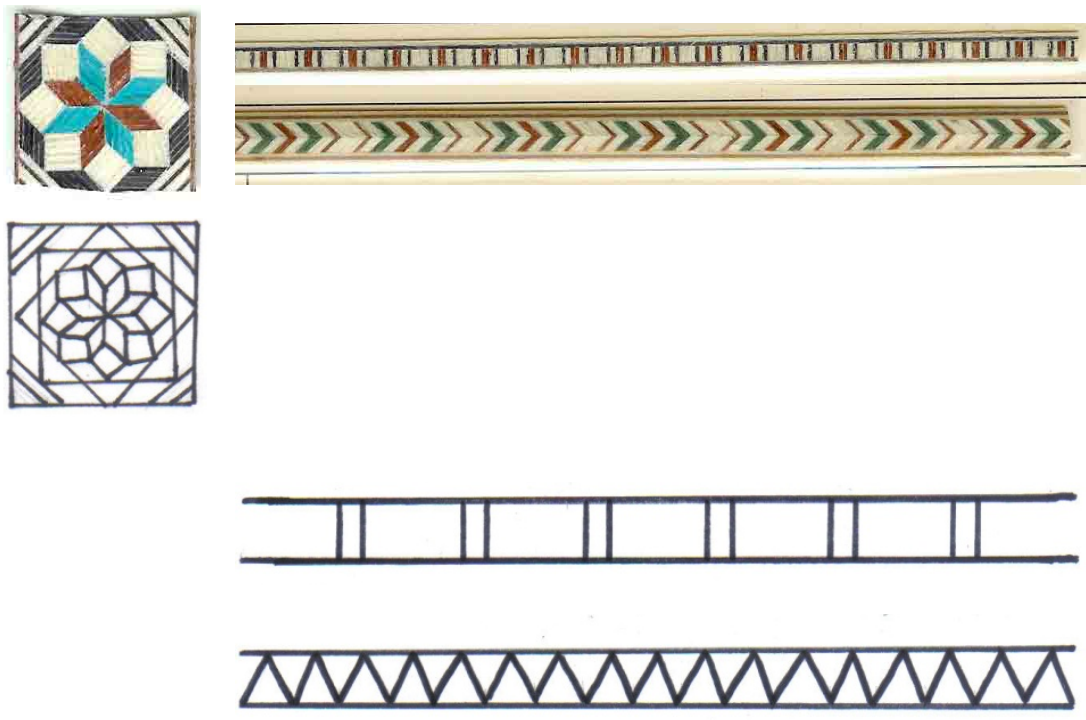

Figura 2. Estrellas y grecas de Taracea reales (parte superior) y modelos (inferior).

- Elaboración del núcleo central del objeto: Recubrir la superficie exterior del objeto, pegando los elementos que han recortado anteriormente, realizando simetrías, seriaciones, etc, dejando total libertad en el diseño.

- Perfeccionamiento y terminación: Pegar aquellos elementos que no estén bien pegados, plastificarlo y pasarlo del plano al espacio; es decir, juntar los 
laterales del rectángulo para formar así un cilindro. Finalmente, para terminar el lapicero, lo uniremos a la tapa de plástico que actuará de base.

El proceso se representa de en la Figura 3.
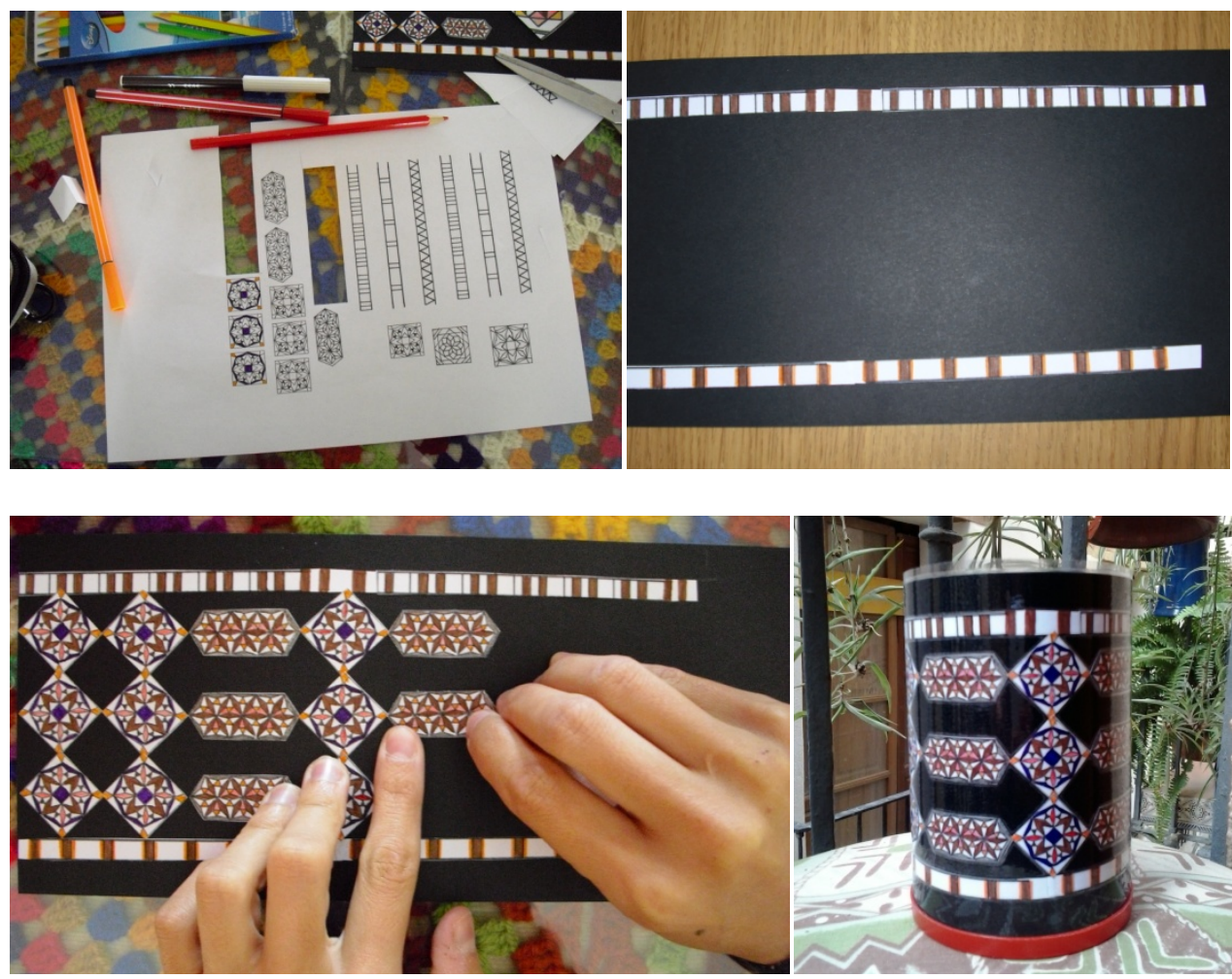

Figura 3. Proceso de elaboración del objeto (lapicero de Taracea cilíndrico).

\section{Evaluación}

Para la evaluación del microproyecto se llevarán a cabo varias actividades. Por un lado, al ir desarrollando cada apartado de lo programado se llevará un protocolo de observación mediante el cual podamos ir comprobando de forma grupal si los niños desarrollan los objetivos deseados. También al finalizar realizaremos algunas actividades de "control" que nos permitan evaluar la adquisición de los objetivos de una forma individual. Con ambos instrumentos de medida podemos obtener información de las dificultades encontradas durante el proceso. A continuación mostramos una de las actividades de control.

\section{Actividad de Control 1}

Esta actividad tiene como fin evaluar de forma individual los objetivos generales de todo el proyecto. Para ello, sería adecuada una actividad de comunicación, correspondiente a la fase final del proceso constructivo, es decir, una vez que han hecho todo el proyecto, tratar de comunicar a los demás aquello que han experimentado. Por lo que hemos creído conveniente que la actividad corresponda al área de expresión. Esta tendrá dos variantes una más sencilla y otra más compleja, dando distintas posibilidades de elección.

Concretamente se les pedirá: 
- Actividad 1 Tipo A: Con papel y lápices de colores realizar un dibujo que represente lo que más les ha gustado de todo el proyecto.

- Actividad 1 Tipo B: Elaboración de un collage con distintos materiales en el que representen el proceso de construcción del lapicero o de lo visto en el taller.

El baremo para valorar estas dos actividades se centrará en los aspectos cualitativos siguientes: el más creativo, completo y el que muestre mejor la realidad de la experiencia vivida. Para valorar el aspecto cuantitativo se clasificaran los trabajos en 3 niveles progresivos de logro de los objetivos.

Teniendo en cuenta la evaluación grupal, podremos ver los errores de programación o implementación del proyecto y los ajustes y desajustes que tienen que ver con las características del grupo. De este modo, podremos valorar mejor las actividades individuales ya que están mediatizadas por el trabajo hecho en grupo.

\section{CONCLUSIONES}

- La dimensión cultural condiciona el pensamiento matemático.

- Las matemáticas son universales en el sentido de que son una parte de todas las culturas pero a la vez son tan diversas como lo son las mismas culturas.

- La didáctica a través del microproyecto, además de la adquisición de conocimiento, implica el desarrollo de habilidades y actitudes.

- El microproyecto permite trabajar las matemáticas desde una perspectiva más cercana a las niñas y niños; facilitando un aprendizaje basado en el constructivismo.

- La técnica artesanal de la Taracea, como objeto de estudio, permite una secuencia didáctica constructivista: acción-reflexión y representación.

\section{BIBLIOGRAFÍA}

Aebli, H. (1979). Una Didáctica Fundada en la Psicología de Jean Piaget. Buenos Aires: Kapeluz.

Alsina, A. (2011). Educación Matemática en contexto: de 0 a 3 años. Barcelona: Horsori

D'Ambrosio, U. (1985). Ethnomathematics and its place in the history and pedagogy of mathematics. For the Learning of Mathematics, 5(1), 44-48.

Carretero, M. (1997). Constructivismo y educación. Méjico: Progreso.

Fernández, J. (2003). Artesanía, Folklore y arte popular. Buenos Aires: Condorhuasi.

Geertz, C. (1988). La interpretación de las culturas. Barcelona: Gedisa.

Hernández, F. (1988). La globalización mediante proyectos de trabajo. Cuadernos de Pedagogía, 155, 54-59.

Oliveras, M.L. (2006). Etnomatemáticas, de la multiculturalidad al mestizaje. En J. M. Goñi (Coord.), Matemáticas e interculturalidad (pp.117-149). Barcelona: Graó

Oliveras, M. L. (2000). Etnomatemáticas. En J. Fuentes y M. L. Oliveras (Ed.), Matemáticas en la sociedad. (pp. 39-46). Granada: Repro Digital. 
Oliveras, M. L. (1996). Etnomatemáticas. Formación de profesores e innovación curricular. Granada: Comares.

Requesens, E. y Díaz G. (2009). Una revisión de los modelos didácticos y su relevancia en la enseñanza de la ecología. Revista Argentina de Humanidades y Ciencias Sociales, 7. 